\title{
SÔBRE UM CENSO DE CRIANÇAS DESAJUSTADAS NAS ESCOLAS PÚBLICAS DO RECIFE
}

\author{
Gonçatives Fernanites * \\ J. J. Aives Tavarfs **
}

O censo de crianças desajustadas nas escolas públicas do Recife, realizado pela Seç̧ão de Ortofrenia e Higiene Mental no ano de 1951, constitui o primeiro empreendimento desta natureza entre as atividades do Departamento de Saúde Pública do Estado de Pernambuco. Cumprindo sua missão de prevenir ao invés de curar, de "conservar normal a criança normal", mas também sem esquecer a face corretiva da Higiene Mental, a Secção de Ortofrenia estudou sob aspecto médico-psicológico um total de 23.057 crianças matriculadas no curso primário dos diversos estabelecimentos oficiais do Recife. Dentre esta população escolar, 2.247 crianças $(9,74 \%)$ foram consideradas desajustadas e, portanto, merecedoras de maiores cuidados por parte de médicos, mestres e pais. $O$ material humano foi estudado sob vários ângulos. Deu-se atenção a características raciais, especialmente cór, idade, classe, situação no currículo escolar e procedência, bem como às características do desajustamento. Para estudo posterior do ambiente escolar recifense determinou-se a percentagem de desajustados com que as diversas escolas concorreram na integração do total de crianças-problemas. Esta pesquisa corresponde a meses de exaustiva labuta e não teria sido possível sem a colaboração dedicada de Sarah Times de Carvalho (funcionária da Assistência a Psicopatas, a cargo de quem ficou o manuseio de mapas e a apuração dos diversos dados aqui registrados) e a cooperação das diretoras de grupos escolares e professoras de escolas isoladas. Expomos, condensados, os resultados. Alguns não nos permitem tirar conclusões por falta de outros dados que os comple. mentem e cuja obtenção independe da nossa vontade.

* Professor da Fac. Ciênc. Méd. de Pernambuco. Chefe da Secção de Ortofrenia e Higiene Mental do Departamento de Saúde Pública do Estado de Pernambuco.

* Assistente da Fac. Ciênc. Méd. de Pernambuco. Assistente voluntário da Secção de Ortofrenia e Higiene Mental. 


\begin{tabular}{|c|c|c|c|}
\hline \multirow{2}{*}{$\mathrm{D}$ a $\mathrm{d}$ o $\mathrm{s} \quad \mathrm{r}$ a $\mathrm{c}$ i a $\mathrm{i} s$} & \multicolumn{2}{|c|}{$S e \times 0$} & \multirow{2}{*}{ Totais } \\
\hline & M & $\mathrm{F}$ & \\
\hline Ieucodermos $\ldots$. & 662 & 513 & 1.205 \\
\hline Faiodermos $\quad \ldots \ldots \ldots \ldots \ldots \ldots \ldots$ & 415 & 372 & 787 \\
\hline Melanodermos $\ldots .$. & 127 & 94 & 221 \\
\hline Sem informes ... & 18 & 36 & 34 \\
\hline
\end{tabular}

Quadro 1

Predominam, entre as crianças desajustadas nas escolas públicas do Recife, as de raça branca; tôdas as raças concorreram com mais crianças do sexo masculino que do feminino (quadro 1). Entretanto, não é possível tirar conclusões quanto à suscetibilidade racial aos fatôres desajustantes, pois, para isto, necessitaríamos de dados percentuais só possíveis se tivéssemos à mão números referentes a tôda a população escolar do Recife, dados êsses inexistentes na Secretaria de Educação.

\begin{tabular}{|c|c|c|c|}
\hline \multirow{2}{*}{ I $d$ a $d$ e $s$ (anos) } & \multicolumn{2}{|c|}{$S \mathrm{e} \times 0$} & \multirow{2}{*}{ To: $a \mathrm{is}$} \\
\hline & $\mathrm{M}$ & F & \\
\hline 4 a $6 \ldots$ & 64 & 28 & 92 \\
\hline 7 a 8 & 239 & 151 & 390 \\
\hline 9 a $10 \ldots$ & 326 & 274 & 600 \\
\hline 11 a $12 \ldots$ & 3333 & 312 & 645 \\
\hline 13 a 14. & 137 & 163 & 300 \\
\hline 15 e mais & 35 & 24 & 59 \\
\hline Sem informes $\ldots \ldots \ldots \ldots \ldots \ldots \ldots$ & 90 & $\tau 1$ & 161 \\
\hline
\end{tabular}

Quadro 2

As maiores cifras correspondem, como se vê, às idades compreendidas entre 9 e 12 anos; as idades de 13 e 14 anos, correspondentes à fase da puberdade (no Nordeste do Brasil), dão, também, cifras expressivas; no sexo masculino sempre as cifras foram maiores (quadro 2 ). 


\begin{tabular}{|c|c|c|c|}
\hline \multirow{2}{*}{ Clas s e s } & \multicolumn{2}{|c|}{$S e \times o$} & \multirow{2}{*}{ Tota is } \\
\hline & $\mathbf{M}$ & $\mathrm{F}$ & \\
\hline Jardim da Infância $\ldots \ldots \ldots \ldots \ldots$ & 38 & 18 & 56 \\
\hline Preliminar $\quad \ldots \ldots \ldots \ldots \ldots \ldots \ldots \ldots$ & 53 & 34 & 87 \\
\hline $1^{q}$ Série $A \quad \ldots \ldots \ldots \ldots \ldots \ldots \ldots$ & 516 & 370 & 886 \\
\hline $1^{\text {q }}$ Série $13 \ldots \ldots \ldots \ldots \ldots \ldots \ldots$ & 29 & 20 & $\$ 9$ \\
\hline 2a Série $\ldots \ldots \ldots \ldots$ & 254 & 259 & 513 \\
\hline $3^{\text {a }}$ Série $\ldots \ldots \ldots$ & 172 & 169 & 34.1 \\
\hline 4ạ Série $\ldots \ldots \ldots$ & 138 & 116 & 254 \\
\hline $5^{q}$ Série $\ldots \ldots \ldots \ldots \ldots \ldots \ldots \ldots$ & 25 & 30 & 55 \\
\hline Sem informes $\ldots \ldots \ldots \ldots \ldots \ldots \ldots$ & 4 & 2 & 6 \\
\hline
\end{tabular}

Quadro :3

A $1^{\text {a }}$ série A dá o maior contingente de crianças excepcionais; sua maioria é constituída de meninas de baixo quociente intelectual, que poderiam obter maior aproveitamento escolar se ensinados por métodos especiais. A $2^{\text {a }}$ série segue-lhe as pègadas. Os extremos apresentam menor número de escolares-problemas. Para assentarmos conclusões de conjunto fazem-nos falta os dados percentuais que reclamamos ao comentar o quadro i. De qualquer forma, demonstram êstes números a necessidade de um estudo amplo e mais acurado do meio escolar, suas necessidades e falhas, quer sob o ponto de vista pedagógico, quer sob o ponto de vista sanitário.

\begin{tabular}{|c|c|c|c|}
\hline \multirow{2}{*}{ Situação no currículo } & \multicolumn{2}{|c|}{$S e \times o$} & \multirow{2}{*}{ Tota i s } \\
\hline & M & F & \\
\hline  & $\begin{array}{r}369 \\
789 \\
90\end{array}$ & $\begin{array}{r}268 \\
663 \\
78\end{array}$ & $\begin{array}{r}637 \\
1.452 \\
158\end{array}$ \\
\hline
\end{tabular}

Quadro 4

\begin{tabular}{|c|c|c|c|}
\hline \multirow{2}{*}{ Proced $\hat{e} n c i a$} & \multicolumn{2}{|c|}{$\mathrm{S}$ e $\mathrm{x} o$} & \multirow{2}{*}{ Totais } \\
\hline & $\mathbf{M}$ & $F$ & \\
\hline $\begin{array}{l}\text { Recife } \ldots \ldots \ldots \ldots \ldots \ldots \ldots \ldots \ldots \\
\text { Interior do Estado } \ldots \ldots \ldots \ldots \ldots \ldots \ldots \\
\text { Sem informes } \ldots \ldots \ldots \ldots \ldots \ldots \ldots \ldots\end{array}$ & $\begin{array}{r}947 \\
24 \\
258\end{array}$ & $\begin{array}{r}779 \\
18 \\
221\end{array}$ & $\begin{array}{r}1.726 \\
42 \\
479\end{array}$ \\
\hline
\end{tabular}


Para o estudo estatístico do desajustamento tomou-se como modêlo as fórmulas empregadas pelo Consultório de Neuro-Psiquiatria da Inspecção Médica Escolar do Consejo Nacional de Educación da República Argentina. O quadro 6 apresenta as diversas formas de desajustamento encontradas, em seus índices numéricos:

\begin{tabular}{|c|c|c|c|}
\hline \multirow{2}{*}{ Formas de desajustamento } & \multicolumn{2}{|c|}{$S$ e $\times o$} & \multirow{2}{*}{ Tota is } \\
\hline & $\mathbf{M}$ & $F$ & \\
\hline Atrasados & 556 & 538 & 1.094 \\
\hline Instáveis & 143 & 47 & 190 \\
\hline Distraídos . & $19 \pi$ & 166 & 363 \\
\hline Nervosos & 111 & 97 & 208 \\
\hline Esquizóides $\quad \ldots$ & 53 & 24 & 77 \\
\hline Com defeito de linguagem .. & 107 & 53 & 160 \\
\hline Com defeito de audição $\ldots .$. & 11 & 13 & 24 \\
\hline Com defeito de visão & 51 & 78 & 129 \\
\hline Mal definido & 1 & 0 & 1 \\
\hline Sem informes $\ldots \ldots \ldots \ldots$ & 0 & 1 & 1 \\
\hline
\end{tabular}

Quadro 6

$\mathrm{O}_{\mathrm{s}}$ atrasados (oligofrênicos?) constituem a maioria (1.094 ou 48,68\%) dos desajustados; seguem-se-lhes os distraídos, os nervosos e os portadores de defeito de linguagem. Entre êstes últimos há a assinalar uma ostensiva predominância entre os indivíduos do sexo masculino.

Estes dados demonstram, a mais, a necessidade do exame médico-psicológico do escolar no ato da matrícula, com a decorrente triagem para classes especiais no caso dos de baixo quociente intelectual, providência que deveria ser extensiva aos portadores de defeitos orgânicos que necessitam de aprendizado corretivo ou especializado em classes separadas.

Estudando a percentagem de crianças com aproveitamento precário verificamos, ao contrário do que era de esperar, que as escolas de menor matrícula forneciam os maiores dados percentuais. Investigamos 72 escolas. Dentre estas, 22 tinham matrículas que se expressavam por dezenas; as 50 restantes tinham matrículas orçando em centenas. A percentagem de desajustados foi, de maneira geral, maior nas primeiras que nas últimas, fenômeno que representamos nos quadros 7 e 8 . 


\begin{tabular}{|c|c|}
\hline Percentagem de desajustados & $\mathrm{N}^{\mathrm{a}}$ de escolas \\
\hline Com $\quad 100 \% \quad \ldots \ldots$. & 1 \\
\hline Mais de $90 \%$. $\ldots \ldots \ldots \ldots \ldots \ldots \ldots \ldots \ldots \ldots \ldots$ & 1 \\
\hline 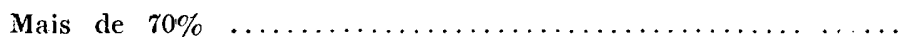 & 1 \\
\hline 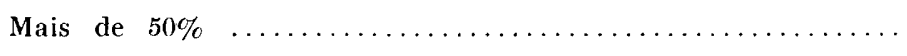 & 1 \\
\hline 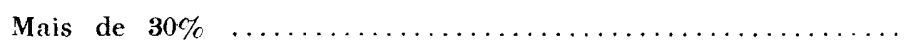 & 2 \\
\hline Mais de $20 \%$ & 5 \\
\hline Mais de $10 \% \ldots \ldots \ldots$ & 3 \\
\hline Total de escolas com mais de $10 \%$ de desajustados. & 14 \\
\hline
\end{tabular}

Quadro 7 - Grupo de 22 escolas com matrícula inferior a 100 alunos.

\begin{tabular}{|c|c|}
\hline Percentagem de desajustados & $\mathrm{N}^{\circ}$ de escolas \\
\hline Mais de $30 \%$.. & 1 \\
\hline Mais de $20 \%$ & 4 \\
\hline Mais de $15 \% \ldots$ & 3 \\
\hline Mais de $10 \% \ldots$ & 18 \\
\hline Total de escolas com mais de $10 \%$ de desajustados. & 26 \\
\hline
\end{tabular}

Quadro 8-Grupo de 50 escolas com matrícula superior a 100 alunos.

Hipóteses várias nos ocorrem diante dêstes quadros. No quadro ? tem-se uma escola com $100 \%$ de desajustados, uma segunda com mais de $90 \%$, uma terceira com mais de $70 \%$ e outra com mais de $50 \%$. Casos de percentagem por agrupamento, no percentual por escola, tão elevados que nos fazem interrogar: o fator desajustante não estará nestas próprias escolas, considerando a elevação global dum único tipo de desajustamento (atrasados)? Não terão sua origem num primo-desajustamento: o das suas próprias professoras?

Quanto ao fato das escolas com matrícula superior a 100 alunos apresentarem menor índice de desajustamento, uma razão provável nos ocorre: estas escolas, pelo seu próprio aglomerado estudantil e formação natural de círculos, equipes, incremento das relações "de igual para igual", teriam agido como uma forma, digamos espontânea, de "cura de comunidade", dando-nos, mercê de fatôres "socializantes" diversos, menores ín- 
dices de desajustamentos. Uma interpretação impessoal dêstes últimos resultados, entretanto, dependerá de mais acurado estudo do meio escolar, empreendimento que ousaremos tentar, mau grado a deficiência em pessoal e material com que luta nosso Serviço desde sua fundação.

\section{RESUMO}

Os autores estudaram, nas escolas públicas da cidade do Recife (Estado de Pernambuco, Brasil), sob o ponto de vista médico-psicológico, 23.057 escolares. Dentre êstes, $2.247(9,74 \%)$ se mostravam desajustados, aberrando do tipo médio. 0 material humano foi apreciado de vários ângulos: raça, idade, série, situação no currículo, procedência e formas de desajustamento. Os autores concluem pela necessidade de mais amplo estudo do meio escolar sob o duplo aspecto sanitário e pedagógico, bem como pela necessidade de exame médico-psicológico no ato da matrícula, visando a constituição de classes homogêneas. Lembram, também, a possibilidade de certo número de desajustamentos ter como causa um primo-desajustamento: o da própria professora.

\section{SUMMARX}

The authors have studied 23,057 school-children in the public schools of Recife (Pernambuco - Brazil) under a psychologic-medical point of view. Among these 2,247 (9.74 per cent) showed themselves maladjusted, out of the medium type. The human material was studied from many angles: race, age, degree, situation in the curriculum, procedence and maladjustements' forms. The authors come to the conclusion that it is necessary more extensive study of the school's ambient under the sanitary and pedagogic aspects, as well as the necessity of a medical-psychological examination at the beginning of the school-year, observing the situation of the homogeneous classes. They remember also the possibility of a certain number of maladjustments having as a cause a prime maladjustment of the school-teacher herself. 
tido foco epileptógeno frontal, explicando perfeitamente sintomatologia de convulsões de tipo bravais-jacksoniano e hemiparesia, independentemente da calcificação tipo angiomatosa, de situação occipital.

\section{SUMMARY}

Electroencephalographic studies in intracranial vascular anomalies.

Reviewing the cases published in the literature and studying his own material (3 cases of Surge-Weber-Dimitri's disease, one case of aneurysm of the medial cerebral artery and 5 cases of arteriovenous fistula) by the clinic, the EEG, the roentgenogram and the arteriogram, the author shows the importance of the EEG record in the establishment of correct diagnosis and in the choice of treatment in these cases.

The author concludes:

1) In the cases of arteriovenous fistula, the EEG showed focal signs in 4 cases; in 2 of them, the EEG record demonstrated the necessity of arteriograms, for the patients have had one and two seizures, respectively, bringing out the possibility of etiological diagnosis and surgical treatment. In another case, the EEG gave orientation for the diagnosis; the 55 year-old patient had been considered as an epileptic for 25 years. In the fifth case (the aneurysm was small and located between parietal convolutions) the EEG did not show focal signs.

2) In the case of aneurysm of the medial cerebral artery, located in the base of the brain, the EEG revealed diffuse and bilateral signs, probably due to the previous subarachnoid hemorrhage.

3) In the 3 cases of Sturge-Weber-Dimitri's disease, the EEG showed records with depressed electrical activity, corresponding to the calcified areas. In one case the EEG also revealed the presence of a frontal focus which explained very well the Bravais-Jacksonian seizures and the hemiparesis, independently of the occipital angiomatous calcification.

\section{BIBLIOGRAFIA}

1. Raskin, N. - Angiomatous malformations of the brain. J. Neuropathol. a. Neurol., 8:326-337, 1948. 2. Craig, M. J. - Encephalo-trigeminal angiomatosis (Sturge-Weber-Dimitri). J. Neuropathol. a. Exper. Neurol., 8:3C5-318, 1949. 3. Busch, E., Broager, B. e Hertz, H. - Electroencephalographically localized focus in case of Sturge-Weber-Krabbe syndrome, extirpated with good result. Acta Psychiat. et Neurol., 24:1-8, 1949. 4. Cornill, L., Paillas, J. e Gastaud, H. - Maladie de Sturge-Weber-Krabbe, traitée par excision corticale. Etude histologique et électroencéphalographique. Ann. Méd.-psychol., 107:304, 1949. 5 . Monnier, M. e Mutrux, S. - Étude électroencéphalographique d'un cas de maladie de Sturge-Weber-Krabbe. Ann. Méd.-psychol., 107:340 (outubro) 1949. 6. Ra- 
dermecker, J. - L'électroencéphalographie dans l'angiomatose encéphalo-trigeminée de Sturge-Weber-Krabbe. Acta Neurol. et Psychiat. Belgica, 51:427-452 (julho) 1951. 7. Silver, M., Taft, .G e Tennant, J. - The electroencephalogram in some angiomatous malformations. EEG. a. Clin. Neurophysiol. J., 4:245 (maio) 1952. 8. Roseman, E., Bloor, B. M. e Schmidt, R. P. - The electroencephalogram in intracranial aneurysms. Neurology, 1:25-38 (janeiro) 1951. 9. Elvidge, A. e Fiendel, H. - Surgical treatment of aneurysm of anterior cerebral and of anterior communicating arteries diagnosed by angiography and electroencepialography. J. Neurosurg., 7:13-32 (janeiro) 1950. 10. Woodhall, 13. e Lowenbach, M. - Congenital cerebral aneurysm localized by electroencephalography. Southern M. J., 36:580-87, 1943. Cit. por G. Lasorthes: Les Hémorragies Intracraniennes, Masson et Cie., Paris, 1952, pág. 165 (Aneurysmes cérébraux). 11. Thiébaut, F., Philippides, D., Rohmer, R. e Montreuil, B. - Angiome intraveineux du cerveau. Rev. Neurol. (julho) 1951. Cit. por Lazorthes, idem 10, pág. 205. 12. Mattos Pimenta, A. - Aspectos neurocirúrgicos das malformações vasculares cerebrais. Resumo in Rev. Paulista de Med., 41:429 (dezembro) 1952.

Rua Barão de Itapetininga, jo, $1^{\text {D }}$ andar - São Paulo. 